\title{
Improving Wireless Access Control Schemes via Adaptive Power Regulation
}

\author{
Francesca Cuomo and Cristina Martello \\ Dpt. Info-Com, University of Rome "La Sapienza" , \\ Via Eudossiana, 18 - 00184 Rome (Italy) \\ \{cuomo, martello\}@infocom . uniroma1.it
}

\begin{abstract}
Two challenging issues for future wireless communication systems are the support of Quality of Service (QoS) and the definition of flexible access schemes to be used in heterogeneous systems (public cellular systems, wireless local area and ad-hoc networks). This paper deals with the class of Radio Resource Control (RRC) based on power regulation, where typical PHY layers parameters (powers, interference, etc.) are combined with upper layers ones to i) increase radio channel utilization; ii) perform admission control of heterogeneous wireless links; iii) maintain negotiated QoS levels expressed as a function of the Signal-toInterference-Ratio (SIR). In the recent literature power control is mainly employed to dynamically adapt the transmission of concurrent links to the varying system conditions (due to channel quality, mobility) still guaranteeing the perceived QoS. In this framework, we investigate how the design of a suitable power control can be used in the support of traffic requiring some QoS guarantees. The paper presents a paradigm where all the RRC operations are performed in a distributed way, i.e., independently for each link involved in a communication. The distributed approach assures that the strategies are quite flexible to be used both in cellular-like systems and in ad-hoc networks.
\end{abstract}

\section{Introduction}

One challenging issue in the design of future wireless communication systems is the possibility to provide Radio Resource Control (RRC) schemes combining, in accordance to a cross-layer approach, PHY, MAC and RLC information in order to optimize the use of the radio resource and satisfy user requirements. This paper deals with the class of RRC based on PoWer Control (PWC) where typical PHY layers parameters (power, interference, etc.) are combined with upper layers ones to increase radio channel utilization and to support Quality of Service (QoS). PWC is the paradigm to accommodate in a wireless system users heterogeneous both in QoS requirements (data rate, packet error rate, etc.) and in perceived quality of the respective wireless channel [1][2][3].

In the recent literature PWC is envisioned as the means to adapt dynamically the transmission of concurrent links to the varying system conditions (due to channel quality and mobility) still guaranteeing the desired QoS. In this framework we investigate how the design of a suitable PWC scheme can be used for 
the support of traffic requiring QoS guarantees that can be expressed, in a very general way, as a soft state of the Signal-to-Interference-Ratio (SIR); i.e., for each link it is negotiated that the SIR will not drop below a given threshold. We remark that the SIR is a simple metric that implicitly includes QoS parameters like the Bit Error Rate (BER), data rate (e.g., processing gain in CDMA systems) and consequently packet transmission time. RRC based on PWC assures that the system resource allocation is adapted to the current environment state.

The paper aims at presenting the frame of PWC exploited for the QoS support in a system where all the RRC operations are performed in a distributed way, i.e., independently for each link involved in a communication. The distributed approach assures that the strategies are quite flexible to be used in both an infrastructured and an ad-hoc network.

The remainder of this paper is organized as follows. Section 2 briefly recalls some literature on distributed power controlled access schemes while in Section 3 we present our proposal (named Distributed Power Regulated Admission Control scheme, D-PRAC); in particular we describe the admission control rules and we discuss issues concerning the adaptivity to system's dynamics as well as implementation aspects. A comparison of D-PRAC with other approaches found in literature is presented by a performance analysis (Section 4). Finally, Section 5 concludes the paper.

\section{Admission Control Schemes Based on Power Control}

Quality of service based on adapting transmission power allows to:

1. establish and maintain links by adapting power levels on the basis of current interference and link extension;

2. make links achieving differentiated QoS levels in terms of rate and target SIR (see for example [4]);

3. mitigate interference and thus channel reuse.

In classical cellular systems (e.g., UMTS) PWC is mainly employed as a mechanism to uniform the power levels of different links concurrent to the same Base Station [5]. Here we extend the PWC adoption to perform the Admission Control (AC) of links requiring some QoS in a wireless network and to maintain the desired QoS during the link lifetime. Furthermore, it can be shown that a suitable PWC can improve the overall system performance and can guarantee an efficient channel reuse that is a key aspect when referring to networks lacking of spectrum (cell) planning schemes.

We focus on distributed AC, where each link will autonomously decide its own admission by collecting information regarding the system it is going to enter. In particular the receiver typically has the task of measuring the perceived interference and reporting the result to the transmitter [6]. Besides measurements, some proposals (e.g., [7]) provide also for signaling broadcasted by each device (typically the transmitter) to all others as further information used to coordinate the power selection process of a number of links. Depending on the 
multiple access technique, PWC schemes can be applied in single channel systems (e.g., 802.11) and in multi-channel ones (e.g., CDMA). However, it can be shown that in both cases the problem of power controlled access can be formalized in very similar terms [8]. The main difference between the two is that, in case of multi-channel, interference is mitigated by a processing gain and thus resource reuse is higher. The need of making a number of links coexist, leads to the conclusion that each one should regulate its own power in order not to disturb too much the other neighbor communications while keeping to preserve its desired SIR. In [9] it has been investigated how the introduction of PWC in the access procedure can enhance the system performance in terms of radio channel utilization efficiency.

Two main classes of distributed power controlled access schemes can be identified:

1. based exclusively on measurements of the surrounding environment;

2. based on both measurements and signaling among different links.

As for methods based just on measurements, proposals in this class are [6][8][10]. The common idea of these works is that a link can extract information on the system just by performing measurements. In particular in [10] a local (hence distributed) probing scheme is developed aiming at discovering admissibility of the new link just by "comparing" interference measurement results before and after activating the transmission of the link: a link evaluates the impact of its entrance on the others (which will adapt to the new interference) and decides whether it is admissible. In [8] it is proposed a distributed PWC algorithm which protects active links with respect to the interference brought by new access attempts. Each link just applies a rule of power updating on the basis of current SIR measure: the algorithm has the desirable feature to guarantee the maintenance of the target SIR during updating. In spite of their advantageous lack of inter-link signaling, a strong limit of such works consists in not considering the power-constrained problem (i.e., radio devices have a bound on the maximum power they can emit). This problem can not be overcome when links operate only on the basis of measurements. A link evaluates the possibility to enter the system on the basis of its own impact on the others and of their consequent reaction, but in a power constrained system this reaction in terms of additional interference can be less due to power saturation of some device. As a consequence the admission decision could wrongly success causing the drop of power saturated links.

Other approaches face and overcome the problem of power constraint by means of explicit inter-link signaling [7][11][12]. The adopted paradigm consists in protecting links by maintaining a margin with respect to the target SIR. This margin is managed in order to allow the admission of new links avoiding to reconfigure (in particular increase) current transmission powers. These strategies result more robust since maintenance of a margin implies that each link can tolerate positive extra interference due to new accesses and also absorbs link quality variations due to "unpredictable" phenomena (e.g., radio channel fluctuations and devices' mobility). Thus a soft $Q o S$ level is maintained since the changed 
link conditions imply SIR variations above the target SIR thanks to the margin. Obviously a tradeoff exists between setting large margins accommodating a high number of soft QoS flows or fixing the margins at very low values thus reducing system flexibility and the number of contemporary flows. In the case of margin adoption, an explicit inter-link signaling is required since a link willing to be established must regulate its transmission power in accordance to the margins of its neighboring links. Margins exchange could be done periodically (like in [7]) or on demand (like in [11]).

A main difference between schemes based only on measurements and schemes based on both measurements and signaling concerns the way of managing powers each time a new link tries to be established: the power management can be either global or incremental [11]. Typically schemes based only on measurements fall into the class of global power management, since all powers are continuously adapted to current interference; anyway not all the proposals can guarantee consistence with the SIR requirements during the updating phase (like in [10]). Instead schemes based on both measurements and inter-link signaling can be either global or incremental although proposals found in literature do not provide for power reconfiguration and are in the class of the incremental approaches.

In this paper we propose a strategy for distributed admission control based on both measurements and inter-link signaling. In order to privilege the robustness of the $\mathrm{AC}$ and the complexity of an eventual implementation still aiming at a flexible and efficient system, we pursue an incremental-like approach with the additional feature of margins reconfiguration during the link lifetime.

\section{A Distributed Power Regulated RRC Based on Margins}

\subsection{Access Model and Problem Statement}

The considered reference model is constituted by a number $N$ of single-hop interfering links. In a general case, either single or multi channel interface, the following expression can be provided for the SIR (denoted as $\gamma$ ) of the $i$-th link:

$$
\gamma_{i}=\frac{P_{i} \cdot g_{i i}}{\sum_{j=1, j \neq i}^{N} P_{j} \cdot g_{i j}+\eta_{i}}
$$

where $g_{i j}$ represents a kind of "cross-correlation effect" between the $i$-th and the $j$-th link. In general $g_{i j}$ is the total path gain form the $j$-th link's transmitter to the $i$-th link's receiver and accounts for the distance attenuation as well as for shadowing and multi-path fading effects; in case of multi-channel (like CDMA) it also includes the processing gain. $P_{i}$ is the $i$-th link's transmitter emitted power and $\eta_{i}$ is the noise power at the $i$-th link's receiver.

In the framework of a power regulated access, the AC problem can be formulated in the following way. Without loss of generality, we assume that the link requirements are expressed in terms of SIR target $\gamma^{T}$ and - in case of CDMA 
systems - of desired rate, this latter in turn implies that some code with a certain processing gain is required for transmission. We consider that $N-1$ links are already active satisfying their QoS requirements. The problem of evaluating the access of an $N$-th link consists in finding, if it exists, a proper set of transmission powers $\left\{P_{i}\right\}_{i=1, \ldots, N}$ such that $\gamma_{i} \geq \gamma_{i}^{T}$ for each link $i=1, \ldots, N$. The problem can be expressed in the following matrix form allowing to define the well-known condition for existence of a feasible solution:

$$
\begin{cases}(\mathbf{I}-\mathbf{F}) \cdot \mathbf{P} & \geq \mathbf{h} \\ \mathbf{P} & \geq \mathbf{0}\end{cases}
$$

where:

- I is the $N \times N$ identity matrix;

- $\mathbf{P}$ is the column vector $(N \times 1)$ of transmission powers;

- $\mathbf{F}$ is a $N \times N$ matrix which depends on the current topology; in particular $F_{i i}=0$ and $F_{i j}=\gamma_{i}^{T} \cdot \frac{g_{i j}}{g_{i i}}$;

- $\mathbf{h}$ is a column vector $(N \times 1)$ and is essentially related to noise powers $\left(h_{i}=\gamma_{i}^{T} \cdot \eta_{i} / g_{i i}\right)$.

The existence of a feasible solution of problem (2) is expressed as a condition for the maximum modulus eigenvalue of $\mathbf{F}, \rho_{F}$, and is $\rho_{F}<1$.

In other terms, the $N$ links can coexist satisfying their QoS requirements depending on the topology they are structured in. If a solution of problem (2) exists, then the minimum power solution (called Pareto-optimal solution) is:

$$
\mathbf{P}^{*}=(\mathbf{I}-\mathbf{F})^{-\mathbf{1}} \cdot \mathbf{h}
$$

which has the property that, with every other solution, all links' power levels are not lower that their corresponding Pareto-optimal levels. In particular any other solution can be expressed as $\mathbf{P}^{*}=(\mathbf{I}-\mathbf{F})^{-\mathbf{1}} \cdot(\mathbf{h}+\epsilon)$ where $\epsilon$ is a column vector of $N$ real positive values.

\subsection{Approach Based on Margins (D-PRAC)}

As stated before, the method we adopt is based on making active links keep a margin with respect to their desired SIR $\gamma^{T}$. In order to maintain this margin we introduce the Maximum Extra Interference (MEI) an active link can sustain still maintaining its SIR not below $\gamma^{T}$. If MEI is greater than 0 the link perceives a SIR above $\gamma^{T}$, while a MEI equal to 0 means that the link operates at the target SIR. The scheme we propose, D-PRAC, proceeds in a step-by-step way by evaluating the access of a new link given a number of already active ones. Once verified the admissibility of the new link, D-PRAC will maintain the links at power levels $\mathbf{P}^{*}<\mathbf{P} \leq \mathbf{P}_{\mathbf{m a x}}^{\mathbf{d v}}, \mathbf{P}_{\mathbf{m a x}}^{\mathbf{d v}}$ being the column vector of the devices' maximum powers. Thus, in general, the scheme will not be able to configure $\mathbf{P}$ at the Pareto-optimal levels. 
The D-PRAC main steps are:

Starting point: $N-1$ links are active at transmission powers $\mathbf{P}^{(\mathbf{N}-\mathbf{1})}$ (where $N-1$ represents the length of the vector) such that they can tolerate some positive MEI level.

Access attempt: the access of the $N$-th link can take place at power $P_{N}$ within a range $\left(P_{\min , N}, P_{\max , N}\right)$ where $P_{\min , N}$ is the minimum transmission power allowing to reach the desired QoS and $P_{\max , N}$ is the maximum transmission power compliant with (i.e., not exceeding) active links' MEIs and with the device maximum power $P_{\max , N}^{d v}$ (the computation of these parameters is explained in Section 3.3). The admission rule compares $P_{\min , N}$ and $P_{\max , N}$ in order to check whether they limit a feasible range of power values.

Power/MEI selection: if the access can take place (i.e. $P_{\min , N} \leq P_{\max , N}$ ), a criterion must be adopted to select the transmission power. Depending on the chosen transmission power, the new link will acquire a certain MEI level as well as it will consume a certain amount of the other links' MEIs. Thus a major component of the admission rule is definitely the power/MEI selection criterion which affects the radio resource utilization efficiency. We recall that the acquired MEI accounts for the device power constraint since represents the maximum additional interference the link could sustain by keeping its power not above $P_{\max }^{d v}$.

Power/MEI Selection Criterion. We introduce a power/MEI selection criterion which aims at: i) adapting power configuration to environment variations; ii) guaranteeing location fairness with respect to access possibility, iii) avoiding block states that could be overcome with a suitable MEI reconfiguration action. Let us consider a sequence of links acquiring an initial MEI at the access. Due to changing conditions (such as link accesses and releases, radio channel variations and mobility) the active links' MEIs will vary dependently also on the reciprocal distances. At a certain time the AC may fail due to some too low - or even null - MEI, nevertheless the considered link might be admissible after having performed a suitable power reconfiguration. While in [11] every new link enters the network acquiring a constant MEI, on the basis of the previous observation, here we introduce a more flexible definition of the margin approach by providing for an access with balanced and partially reconfigurable MEIs.

Here we deal with the problem of selecting the transmission power of a new $N$ th link within the existing range $\left(P_{\min , N}, P_{\max , N}\right)$. The main point is the tradeoff existing between the new link's and the already active ones' MEIs. The larger the new link's transmission power the larger the MEI it acquires and the larger the other links' MEI consumption. This trade-off can be graphically represented by the plot of MEIs as functions of power $P_{N}$, in particular in Figure 1 we show the MEIs of two active links ( $i$-th and $j$-th) and the $N$-th attempting the access. We observe that $P_{\min , N}$ corresponds to the minimum power above which a non-negative $\mathrm{MEI}_{N}$ is acquired while the value $P_{\max , N}$ is the one annulling the first MEI in the system. The optimal working point consists in the power $P_{\text {opt }, N}$ allowing to maximize the minimum MEI which indeed constitutes a bottleneck 
for further accesses. In fact, the greater the maximum allowed power the higher the probability of a successful access for a further link. Thus an unbalanced MEI configuration would imply that at least one large MEI could be reduced in favor of links with less MEIs.

Another main observation regards the fact that not only MEIs' balancing but also their common value impacts the possibility of accessing the network. Let us assume that all MEIs are equal and that a new link tries to enter the network. Then, no matter the reciprocal link distances, there is enough room for the new link only if enough MEI has been acquired to absorb the new entrance. Since during lifetime of the network MEIs will generally evolve to unbalanced values and will be also consumed by new accesses, a MEI reconfiguration process is introduced in order to partially re-balance the system. According to the incremental philosophy such reconfigurations are not global but partial, i.e., they are locally performed by a subset of links (possibly only one at time) thus resulting in sub-optimal power configurations although they are very simple to be implemented. The general structure of the reconfiguration process is defined by the following steps:

Starting point: $N$ links are active at transmission powers $\mathbf{P}^{(\mathbf{N})}$.

Link selection: according to a suitable criterion, a link - say the $k$-th link $(1 \leq k \leq N)$ - is selected for MEI reconfiguration.

Power/MEI reconfiguration: since the $k$-th link is already active, there actually exists a range of suitable powers $\left(P_{\min , k}, P_{\max , k}\right)$ and it is currently adopting a transmission power $P_{c u r r, k}$ within this range. On the basis of current other links' MEIs it computes and selects for transmission the optimal power $P_{o p t, k}$ which maximizes the minimum MEI. This situation is described in Figure 2 where it is assumed that the $k$-th link corresponds to the one currently perceiving the maximum MEI. This power reconfiguration process is simple since it is performed within MEIs' constraints. As for the criterion of choice of the link to be reconfigured, it actually represents an open point and affects effectiveness of the admission control scheme. In general we can observe that, like in the previous example of Figure 2, reduction of higher MEIs results in improvement of access possibility since it implies a power decreasing which in turn results in an increasing of lower MEIs (e.g., MEI $_{j}$ in the figure).

\subsection{D-PRAC Protocol and Implementation Issues}

To clarify the D-PRAC mechanism, we specify some implementation issues. First we show how the parameters $P_{\min }, P_{\max }, P_{\text {opt }}$ are computed and what information they are derived from. Then we provide the signaling exchange needed for the support of D-PRAC and address the main problems concerning its implementation. $P_{\min }$ is the minimum power that allows the link to reach the desired QoS level and can be computed by imposing that exactly the SIR target is achieved: 


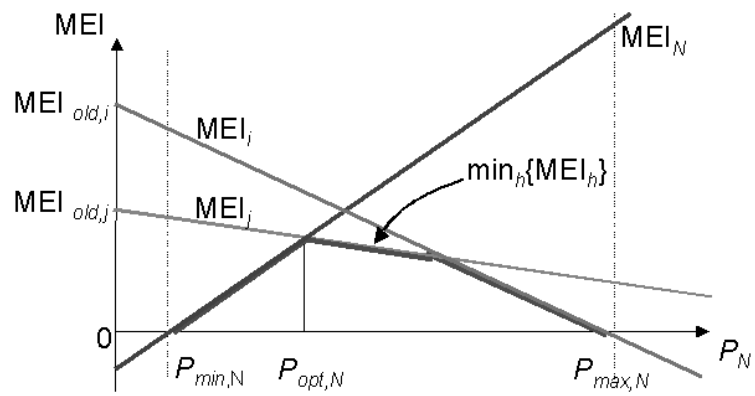

Fig. 1. MEIs as functions of the new link's transmission power.

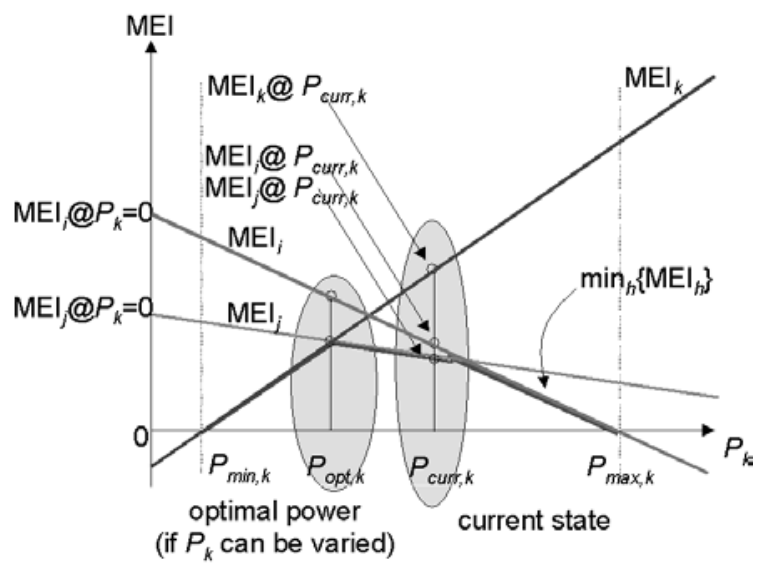

Fig. 2. MEIs as functions of an active link's transmission power under reconfiguration.

$$
\frac{P_{\min , N} \cdot g_{N N}}{\sum_{j=1}^{N-1} P_{j} \cdot g_{N j}+\eta_{N}}=\gamma_{N}^{T} \Rightarrow P_{\min , N}=\gamma_{N}^{T} \cdot \frac{\sum_{j=1}^{N-1} P_{j} \cdot g_{N j}+\eta_{N}}{g_{N N}}=\gamma_{N}^{T} \cdot \frac{I_{N N}}{g_{N N}}
$$

Thus, besides $\gamma_{N}^{T}, P_{\min , N}$ essentially depends on the interference level $I_{N}$ at the receiver and the link extension. The link's receiver shall measure the first quantity and estimate the second one by cooperation with the relevant transmitter, as we will specify in the following.

As for $P_{\max , N}$, it represents the maximum power that at most annuls one of current MEIs and is calculated according to the following expression (where also the device power constraint is considered):

$$
P_{\max , N}=\min \left\{P_{\max , N}^{d v}, \min _{j \neq N}\left\{\frac{M E I_{j}}{g_{j N}}\right\}\right\}
$$

According to (5), $P_{\max , N}$ can be derived if neighbor links' MEIs are known as well as their receivers' distance from the link's transmitter. As for the optimal 
power $P_{o p t, N}$, the new $N$-th link can calculate it on the basis of MEIs and the interference measured $I_{N}$ :

$$
P_{o p t, N}=\min _{i \neq N}\left\{\frac{M E I_{i}+I_{N}}{g_{i N}+g_{N N} / \gamma_{N}^{T}}\right\}
$$

A major component of the mechanism is the signaling of MEIs which must be updated by links' receivers as their variations come about and broadcasted to neighbor transmitters. To this end, two basic methods can be adopted: a periodic broadcasting and an on-demand signaling. Besides, the MEI level can be either explicitly signaled by a data packet or conveyed by a tone whose power level is inversely proportional to MEI (see [7]). In the following we assume a periodic broadcasting. The protocol main steps performed by two radio entities $A$ and $B$ willing to communicate respectively as transmitter and receiver, are the following (qualitatively described in Figure 3):

step $1 A$ contacts $B$ by sending a specific message; the reception of this message triggers in $B$ interference measurements and allows $B$ to estimate the path gain by comparing transmitted and received power relevant to this message; moreover, in the contact message, $A$ includes the desired SIR so that $B$ can compute $P_{\text {min }}$ in accordance to (4).

step $2 B$ notifies to $A$ the minimum power $P_{\text {min }}$.

step 3 In the meanwhile $A$ listens to and collects broadcasted MEIs from neighboring devices. In case of explicit data packets are adopted, they must also include the used transmitted powers so that $A$ can estimate the path gain between itself and the various receivers. In case of tones, $A$ will directly extract the quantity $\frac{M E I_{i}}{g_{A i}}$ from the $i$-th tone's received power. Thus $A$ calculates the maximum power $P_{\max }$ as in (5).

step $4 A$ compares $P_{\text {min }}$ and $P_{\max }$ : if $P_{\min } \leq P_{\max }$ the access can take place and the transmission power $P_{\text {opt }}$ is selected according to the above balancing criterion on the basis of the knowledge of MEIs.

step $5 A$ acknowledges to $B$ the access result and in case of success it notifies the selected transmission power. $B$ calculates its own MEI and signals to neighbors the relevant level.

As transmission starts, the other links' receiver will update their MEIs. A crucial point concerns MEI signaling. In particular the QoS assurance could be affected by not complete and/or inaccurate acquisition of MEIs values; in this case estimation of $P_{\max }$ would be incorrect and the new link could select a transmission power exceeding some MEI and thus causing the relevant drop. Another critical point arising in defining D-PRAC implementation is represented by the problem of concurrent access/reconfiguration attempts. As for access, if two links evaluating the admission rule on the basis of the acquired MEIs succeeded the access procedure at the same time, they might cause an amount of interference level overdoing current MEIs. In a soft QoS context these two events can be overcome by making links tolerate additional quality variations included in a negotiated range. Finally, the selection of MEI when no other links 

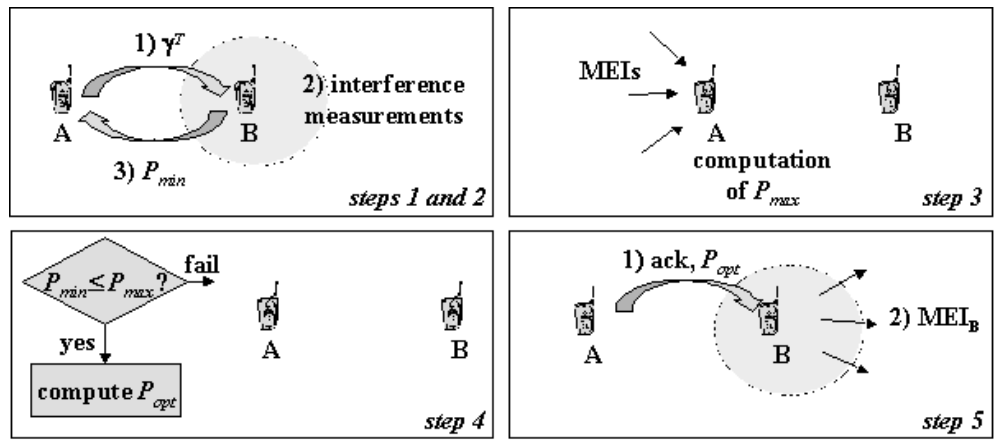

Fig. 3. Main steps of the D-PRAC scheme.

are active (say MEI0) must be specified. In general the selection of MEI0 affects the amount of available resource for new subsequent accesses. Thus a criterion of choice of MEI0 could be to set it at the value allowing to accommodate a given amount of communications before a block occurs referring to an expected situation for further access requests. More in detail the first link should select a power $P_{1}$ depending on the MEI0 by:

$$
\frac{P_{1} \cdot g_{11}}{M E I 0+\eta_{1}}=\gamma_{1}^{T}
$$

MEI0 should be calculated in order to sustain up to a given number N0 with a given $\gamma^{T}$. The estimation can be done only by referring to the expected value of the path gain $\left(g_{e x}\right)$ and accounts for the maximum power allowed to each new link which is equal to $\frac{M E I 0}{N 0 g_{e x}}$.

$$
\frac{g_{e x} \frac{M E I 0}{N 0 g_{e x}}}{\eta_{0}+g_{e x} \cdot P_{1}}=\gamma^{T}
$$

Thus $P_{1}$ and MEI0 are computed as solutions of the equation system constituted by (7) and (8); due to the power constrain the actual adopted $P_{1}$ will be the minimum between $P_{\max }^{d v}$ and the computed solution and the resulting MEI0 can be found by equation (7).

\section{Performance Results}

\subsection{General Behavior of Access Schemes Based on Margins}

We aim at showing the impact of a given MEI configuration for a set of active links on the probability of new access success.

We considered a topology of $N$ links for different values of $N$ and we set their transmission parameters so that they acquired given MEI levels. In particular we considered a square area of $100 \mathrm{~m} \times 100 \mathrm{~m}$, a central frequency of $1 \mathrm{GHz}$, a 


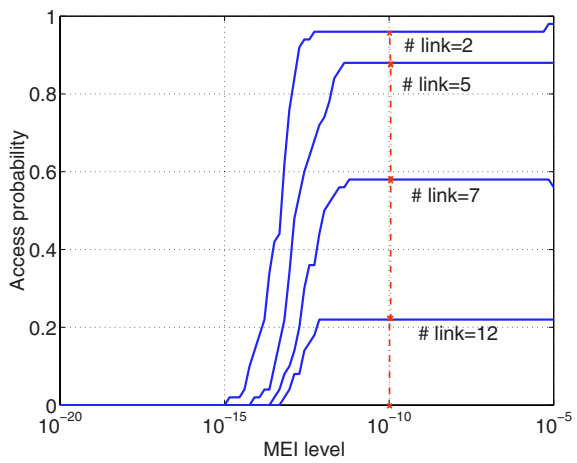

Fig. 4. Access probability as a function of a common MEI level.

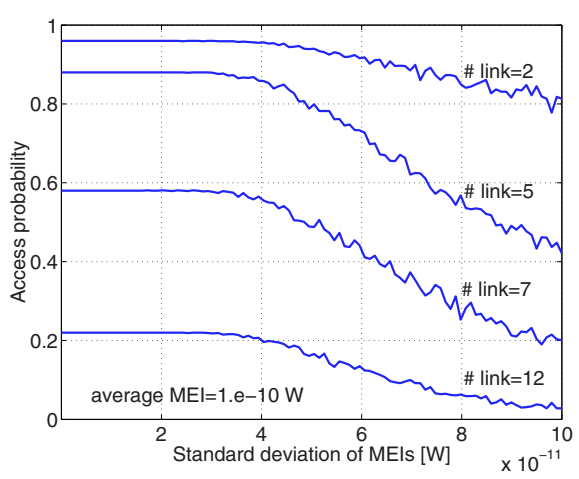

Fig. 5. Access probability as a function of the MEI standard deviation.

geometric factor of attenuation equal to 2 , a processing gain equal to $200, \gamma^{T}=5$, $P_{\max }^{d v}=1 \mathrm{~W}$ and $\eta=2 \mathrm{e}-12 \mathrm{~W}$.

Figure 4 shows the results relevant to the access probability for a new link obtained when a common MEI level (reported in the abscissa) is set in all the active links. Different numbers of active links are considered. The main observation concerns the behavior of the access probability as a step function of the MEI level. Active links have to maintain enough MEI in order to permit the access to a new link. As we try to force a MEI level above the step point, the access probability saturates due to the existence of maximum transmission power reflecting in an upper bound on the acquirable MEI. The access probability saturation level gets lower as the number of active links increases due to the greater link density in the considered area.

Figure 5 reports the access probability obtained when active links' MEI levels are set according to a fixed average value, equal to 1.0 e-10 W highlighted also in Figure 4, and with a variable standard deviation for different values of the number of links. This plot shows the interesting behavior that a MEI configuration with sparse MEI levels degrades the access probability for new links and thus confirms that a margin based strategy aiming at balancing MEIs will outperform schemes which do not care of keeping MEIs balanced.

\subsection{Comparison Among Different Access Strategies}

We first present an example showing the advantage and difference of the proposed AC with respect to the scheme of constant initial MEIs proposed in [11] (here named Constant MEIs approach - C-MEI). As for D-PRAC, in our simulations we did not introduced the power reconfiguration process. Let us consider a sequence of five links willing to activate. The MEIs in correspondence of the receiver of each active link are shown (the values are normalized with respect to MEI0). Table 1 and 2 refer respectively to D-PRAC and C-MEI. Note that a MEI equal to infinite characterizes a non-active link. In particular the considered 
Table 1. Example of 5 links activated by Table 2. Example of 5 links activated by applying D-PRAC. applying C-MEI.

\begin{tabular}{|c|c|c|c|c|c|}
\hline step & $M E I_{1}$ & $M E I_{2}$ & $M E I_{3}$ & $M E I_{4}$ & $M E I_{5}$ \\
\hline $\mathbf{a}$ & 1 & $\infty$ & $\infty$ & $\infty$ & $\infty$ \\
\hline $\mathbf{b}$ & 0.78 & 0.78 & $\infty$ & $\infty$ & $\infty$ \\
\hline $\mathbf{c}$ & 0.68 & 0.77 & 0.72 & 0.68 & $\infty$ \\
\hline $\mathbf{d}$ & 0.37 & 0.76 & 0.71 & 0.67 & 0.37 \\
\hline
\end{tabular}

\begin{tabular}{|c|c|c|c|c|c|}
\hline step & $M E I_{1}$ & $M E I_{2}$ & $M E I_{3}$ & $M E I_{4}$ & $M E I_{5}$ \\
\hline $\mathbf{a}$ & 1 & $\infty$ & $\infty$ & $\infty$ & $\infty$ \\
\hline $\mathbf{b}$ & 0.74 & 1 & $\infty$ & $\infty$ & $\infty$ \\
\hline $\mathbf{c}$ & 0.58 & 0.99 & 0.99 & 1 & $\infty$ \\
\hline $\mathbf{d}$ & $\mathbf{0}$ & 0.98 & 0.98 & 0.98 & 0.71 \\
\hline
\end{tabular}

topology is represented at four steps corresponding to the access of the first link (a), of the second (b), of the fourth (c), and of the fifth (d). In case D-PRAC is adopted (Table 1), the entering link acquires a normalized MEI equal to the minimum one (see for example link 4 with respect to link 1 in (c)). Instead, in case of C-MEI (see Table 2), each new link enters acquiring a constant maximum MEI, i.e., MEI0. Thus at the step (b), with D-PRAC, the two links achieve equal MEIs while with C-MEI the second achieves a normalized MEI equal to 1 causing the maximum reduction on MEI of link 1 . In Table 2 at step (d) the previous unbalanced MEI condition leads to a block of the network for future accesses since link 1's MEI reduces to zero due to the access of link 5 at the maximum power. The disadvantage of unbalanced MEIs is evident in the fact that the other links 2, 3, 4 and 5 continue maintaining useless high MEIs while their reduction would increase MEI of link 1 and unblock the network.

We generated a number of topologies with $N$ links, for different values of $N$, randomly located in a square area of $100 m \times 100 m$, in order to estimate the probability of successfully activating all the links. The adopted parameters are the same as in the previous section. Furthermore, we do not introduce devices' mobility.

We have investigated the impact of the choice of MEI0 on performance for both algorithms D-PRAC and C-MEI. In Figure 6 the probability of activating $N$ links is reported as a function of MEI0 for different values of $N$. Two opposite effects arise: on one hand higher MEI0 values increase the successful probability (first part of the curve), on the other hand (second part of the curve) links acquire higher MEIs thus generating higher interference that future links will have to overcome with higher transmission powers, and this avoids further improvement of the access probability. The saturation of the two curves is due to the maximum transmission power which bounds the maximum initial MEI that can be actually acquired. The behavior in case of either D-PRAC or C-MEI is slightly different since in case of D-PRAC the curve settles in faster than C-MEI. This effect is due to the fact that as concerns D-PRAC MEI0 is just the initial MEI acquired by the first link while then the MEIs evolve according to the balanced mechanism shown in Figure 1] while, as far as C-MEI is concerned, MEI0 represents the initial maximum extra interference tolerated by each entering link. Figure 6 also suggests a possible criterion of reconfiguring MEIs in D-PRAC. When the network evolves towards values of MEIs located in the first part of the curve, the reconfiguration process should make it move to higher MEIs allowing to achieve a 


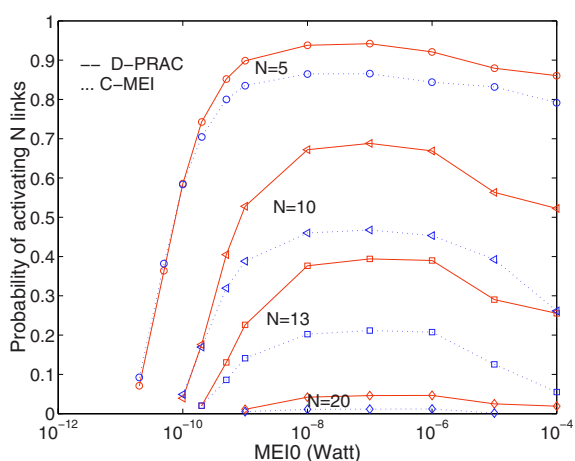

Fig. 6. Probability of activating $N$ links as a function of MEI0.

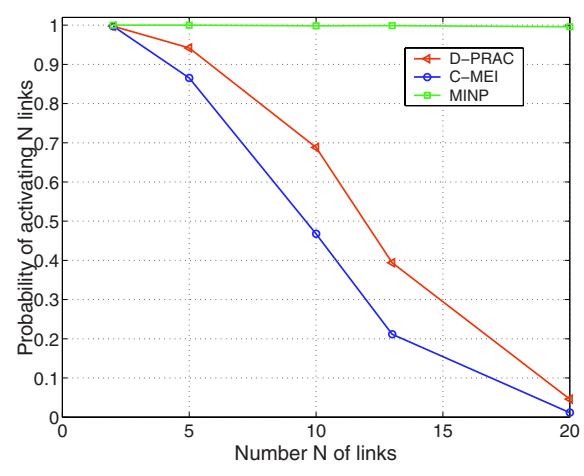

Fig. 7. Probability of activating $N$ links as a function of $N$.

near-maximum access probability for future links. Obviously the problem stands in identifying the working point corresponding to the "elbow" of the curve.

We compared the D-PRAC approach to an ideal scheme reconfiguring powers to the Pareto-optimal solution, i.e. the minimum power (in the following MINP) that can be assumed as reference for the performance analysis. We assume that this scheme behaves ideally rejecting links that would force some link's drop due to the maximum power saturation. The obtained results are reported in Figure 7 for the three strategies D-PRAC, C-MEI and MINP. The MEI0 value for DPRAC and C-MEI is 1.0 e- $8 \mathrm{~W}$. The MINP curve sets the maximum probability that can be achieved by any strategy. We can observe that D-PRAC outperforms C-MEI; besides, since we have not introduced any reconfigurable mechanism yet, we can expect further improvement making D-PRAC curve closer to MINP when one or more links undertake a reconfiguration. When comparing D-PRAC and CMEI, let us remind that the two strategies require the same kind and amount of extra information and thus of signaling overhead. Instead, as for the application of MINP in a distributed scenario, some proposals exist based on probing the radio channel before and after the access (see [10]); however in this case the algorithm neglects the bound on the transmission power of a device implying drop of active links.

\section{Conclusions}

In this paper, we deal with a Radio Resource Control based on distributed power regulation. The system supports QoS flows requiring a given SIR level. Our approach is based on the maximum extra interference that can be tolerated by a QoS flow and can be applied in a distributed system where the optimal "minimum power approach" seems not applicable due to the high complexity required to reconfigure the links. D-PRAC is quite general and may be applied in "CDMA" oriented systems based on multichannel-multirate paradigms. Unlike previous proposals, our margin-based approach allows balancing links' 
parameters to reduce system block probability, typical of incremental admission control approaches. We discuss the advantages of the balanced margin approach by means of qualitative examples and selected performance results. Finally, we give some guidelines for the design of an RRC protocol, which needs only the exchange of few parameters over broadcast channels.

\section{References}

1. T.S. Rappaport, A. Annamalai, R.M. Buehrer and W.H. Tranter, "Wireless communications: past events and a future perspective", IEEE Communications Magazine, vol. 40, no.5, pp. 148-161, May 2002.

2. A. Chandra, V. Gummalla and J.O. Limb, "Wireless Medium Access Control Protocols", IEEE Communications Surveys, Second quarter 2000, pp. 2-15, 2000.

3. N. Bambos and S. Kandukury, "Power-controlled multiple access schemes for nextgeneration wireless packet networks", IEEE Wireless Communications, pp. 58-64, June 2002.

4. D. Kim, "Rate-Regulated Power Control for Supporting Flexible Transmission in Future CDMA Mobile Networks", IEEE J. Select. Areas Commun., vol. 17, no. 5, pp. 968-977, May 1999.

5. D.M. Novakovic and M.L. Dukic, "Evolution of the Power Control Techniques fo DS-CDMA toward 3G Wireless Communication Systems", IEEE Communications Surveys, Fourth Quarter 2000, pp. 2-15, 2000.

6. M. Xiao, N.B. Shroff and E.K.P. Chong, "Distributed Admission Control for PowerControlled Cellular Wireless Systems", IEEE/ACM Transactions on Networking, vol. 9, no. 6, pp. 790-800, December 2001.

7. J.P. Monks, V. Bharghavan and W.W. Hwu, "A Power Controlled Multiple Access Protocol for Wireless Packet Networks", Proc. INFOCOM 2001, pp. 219-228, 2001.

8. N. Bambos and S.C. Chen, "Channel Access Algorithms with Active Link Protection for Wireless Communication Networks with Power Control", IEEE/ACM Trans. On Networking, vol. 8, no. 5, pp. 583-597, October 2000.

9. C. Martello and D. Bocchetta, "Power Controlled MAC Protocols for Wireless Ad-Hoc Networks", Proc. of European Wireless 2002, pp. 319-326, February 2002.

10. C. Zhu and M.S. Corson, "A Distributed Channel Probing Scheme for Wireless Networks", IEEE INFOCOM 2001, pp. 403-411, 2001.

11. S. Lal and E.S. Sousa, "Distributed Resource Allocation for DS-CDMA-Based Multimedia ad hoc Wireless LAN's", IEEE J. Select. Areas Commun., vol. 17, no. 5, pp. 947-967, May 1999.

12. S. L. Wu, Y.C. Tseng, J.P. Sheu, "Intelligent Medium Access for Mobile Ad-hoc Networks with Busy Tones and Power Control", IEEE J. Select. Areas Commun., vol. 18, no. 9, pp. 1647-1657, September 2000. 